Bio - grafia. Escritos sobre la Biología y su Enseñanza. ISSN 2027

Edición Extraordinaria. p.p. $1645-1655$

Memorias del VIII Encuentro Nacional de Experiencias en Enseñanza de la Biología y la Educación Ambiental. III Congreso Nacional de Investigación en Enseñanza de la Biología.

\title{
CATEGORIZACIÓN DE LAS IDEAS PREVIAS DE LOS ESTUDIANTES DE GRADO SÉPTIMO SOBRE EL CONCEPTO DE RESPIRACIÓN ANIMAL
}

\author{
CATEGORIZATION OF THE PREVIOUS IDEAS OF THE STUDENTS IN 7TH GRADE \\ ABOUT THE ANIMAL RESPIRATION CONCEPT.
}

Gil Bohórquez, Bayron ${ }^{1}$

\section{Resumen}

B

I

0

$P$

0

$\mathrm{N}$

E

$\mathrm{N}$

C

I

$A$

La escuela es un escenario de transición desde las ideas previas de los estudiantes hacia formas de comprensión más cercanas a las del conocimiento científico, por ello las ideas previas constituyen el punto de partida más importante en el diseño y la implementación de toda Unidad Didáctica basada en el enfoque de aprendizaje significativo. Se analizaron las ideas previas de 40 estudiantes del grado séptimo (11-15 años) de la Institución Educativa Departamental San Gabriel, ubicado en el municipio de Cajicá, Cundinamarca; a través de la aplicación de un instrumento fundamentado en una pregunta abierta ¿Cómo respiramos? la creación de un mapa conceptual acerca de la respiración en los animales, y un cuestionario con preguntas de selección múltiple y de tipo Falso o Verdadero que permiten conocer lo que el estudiante sabe acerca del concepto de respiración animal. Por último, se realiza la sistematización de datos para un posterior análisis cualitativo de las ideas previas utilizando el programa NVivo 10. Las ideas previas de los estudiantes acerca del concepto de Respiración Animal se asocian principalmente al proceso de intercambio gaseoso. Se observan dificultades para explicar procesos a nivel celular y de tipo estructura-función; aunque los estudiantes evidencian la importancia de la respiración para los animales y su relación con el sistema circulatorio. Es posible categorizar las ideas previas en los modelos explicativos: Intercambio gaseoso, vitalismo, pseudomolecular, y órganos-estructuras.

Palabras clave: Respiración animal, ideas previas, unidad didáctica

\section{Abstract}

The school is a stage of transition from the previous ideas of students to scientific knowledge, previous ideas are the most important point in the design and the implementation of all didactic units based in the significant learning. We analyze the previous ideas of 40 students (11-15 years) in the Educational Institution San Gabriel of Cajicá, Cundinamarca; through the application of an instrument based on an question: How to breathe?, conceptual maps about the animal respiration, and a questionnaire with 
Bio - grafia. Escritos sobre la Biología y su Enseñanza. ISSN 2027

Edición Extraordinaria. p.p. 1645-1655

Memorias del VIII Encuentro Nacional de Experiencias en Enseñanza de la Biología y la Educación Ambiental. III Congreso Nacional de Investigación en Enseñanza de la Biología.

multiple answers and questions of type true or false. Finally, we organize the information to execute a qualitative analysis using the software NVivo 10. The previous ideas of the students about the animal respiration are associated to gas exchange process. The previous ideas are categorized in the explicative models: Gas exchange, vitalism, pseudomolecular, and organs-structures.

Key words: Animal respiration, previous idea, didactic unit.

\section{Introducción}

En ámbitos de la enseñanza de las ciencias es frecuente encontrarse con una gran variedad de respuestas que pretenden explicar determinados hechos, propuestas que parten de los conocimientos y vivencias previas de los estudiantes. En este panorama, algunos investigadores sugieren que las ideas de los estudiantes tienden a ser incoherentes, inconsistentes, fragmentarias y transitorias (Claxton, 1984); y otros consideran que los estudiantes pueden construir esquemas explicativos alternativos que funcionan como teorías, es decir, son consistentes, coherentes y aplicables en amplios períodos de tiempo y a una variedad de fenómenos (Driver et al, 1985). Como señala Driver (1986), estas nociones deben constituir un punto de referencia importante al planificar las tareas de aprendizaje, por ello es importante considerar las ideas previas como el factor más importante que influye en el proceso de enseñanza-aprendizaje (Ausubel, 1968), y serán el punto de partida para el diseño, la implementación y sistematización de toda Unidad Didáctica basada en el enfoque de aprendizaje significativo.

Ausubel postula que el aprendizaje implica una reestructuración activa de las percepciones, ideas, conceptos y esquemas que el estudiante posee en su estructura cognitiva, y concibe al alumno como un procesador activo de la información, dice que el aprendizaje es sistemático y organizado (Díaz y Hernández, 1999). Basándonos en los postulados de Ausubel, la psicología educativa debe concentrarse en la naturaleza y la facilitación del aprendizaje de la materia de estudio y eso significa prestar atención, por una parte, a aquellos conocimientos provenientes de la psicología que hacen falta para dar cuenta de dichos procesos; $y$, por otra, a aquellos principios y premisas procedentes de las teorías de aprendizaje que pueden garantizar la significatividad de lo aprendido, sin que ni unos ni otros se constituyan en fines en sí mismos, ya que lo que realmente interesa es que se logre un aprendizaje significativo en el entorno escolar (Rodríguez et al., 2008). Según Pozo (2006), el aprendizaje significativo se producirá cuando se relacione información nueva con algún concepto inclusor ya existente en la estructura cognitiva del individuo que resulte relevante para el nuevo material que se intenta aprender (Garzón \& Gamboa, 2010). 
Bio - grafia. Escritos sobre la Biología y su Enseñanza. ISSN 2027

Edición Extraordinaria. p.p. 1645-1655

Memorias del VIII Encuentro Nacional de Experiencias en Enseñanza de la Biología y la Educación Ambiental. III Congreso Nacional de Investigación en Enseñanza de la Biología.

El estudio y aprendizaje de la respiración exige el conocimiento de un sinnúmero de conceptos y la instauración de relaciones directas $e$ indirectas con otros procesos corporales y celulares. A través de una síntesis sobre los estudios didácticos actuales en el campo conceptual de la respiración, se destacan estudios relacionados con propuestas de investigación encaminadas a establecer los conceptos centrales que han orientado la enseñanza del campo conceptual de la respiración en los diferentes niveles de escolaridad y las concepciones de los estudiantes sobre la respiración. Banet \&Núñez (1990) han estudiado los esquemas conceptuales de los alumnos sobre este concepto y evidencian la tendencia de los estudiantes al referirse a la respiración como un proceso de intercambio de gases; Núñez \& Banet (1996) se centran en el establecimiento de modelos conceptuales de los estudiantes sobre respiración, digestión y circulación, y las relaciones que se dan entre ellos, destacan el predominio de los modelos conceptuales no relacionados, caracterizados por no identificar adecuadamente el papel del sistema circulatorio en los procesos de respiración y digestión. Y en los estudios de Tamayo (1999) se dedica al reconocimiento de los modelos mentales de los estudiantes sobre respiración. Tamayo et al. (2008) identifican los diferentes modelos que usan los estudiantes para explicar el concepto de respiración (vitalista, teleológico, interrelación vitalista-teleológico, intercambio de gases, sistémico, combustión, oxidación y pseudomolecular) y reconocen posibles obstáculos en el aprendizaje del concepto de respiración (Tamayo et al, 2008). La investigación tiene como objetivo principal categorizar y caracterizar las ideas previas que tienen los estudiantes acerca del concepto de respiración animal para el posterior diseño de la Unidad Didáctica basada en el enfoque de aprendizaje significativo.

\section{Metodología}

Se analizaron las ideas previas de 40 estudiantes del grado séptimo (11-15 años) de la Institución Educativa Departamental San Gabriel, ubicado en el municipio de Cajicá, Cundinamarca. Previamente a la creación de un escenario de transición desde las ideas previas de los alumnos hacia formas de comprensión más cercanas a las del conocimiento científico, se incorpora al proceso un instrumento compuesto por la formulación de una pregunta abierta ¿Cómo respiramos?, la creación de un mapa conceptual acerca de la respiración animal basado en la teoría del aprendizaje de Ausubel-Novak (1978), considerando al mapa conceptual como una representación visual de la jerarquía y las relaciones entre conceptos de cada estudiante. De la misma manera, un cuestionario con preguntas de selección múltiple y de tipo Falso o Verdadero basado en la propuesta de Banet y Núñez (1990) se aplican de manera individual, ya que permiten conocer lo que el 
Edición Extraordinaria. p.p. 1645-1655

Memorias del VIII Encuentro Nacional de Experiencias en Enseñanza de la Biología y la Educación Ambiental. III Congreso Nacional de Investigación en Enseñanza de la Biología.

estudiante sabe acerca del concepto de respiración animal. Por último, se realiza la sistematización de datos para un posterior análisis cualitativo de las ideas previas utilizando el programa NVivo 10, que conlleven a la categorización, caracterización, reflexión, y posterior actuar en el diseño y la implementación de la Unidad Didáctica basada en el enfoque de aprendizaje significativo (Ausubel, 1968).

Figura 1. Mapa conceptual (Instrumento para la recolección de ideas previas) creado por estudiante acerca del concepto de respiración animal.

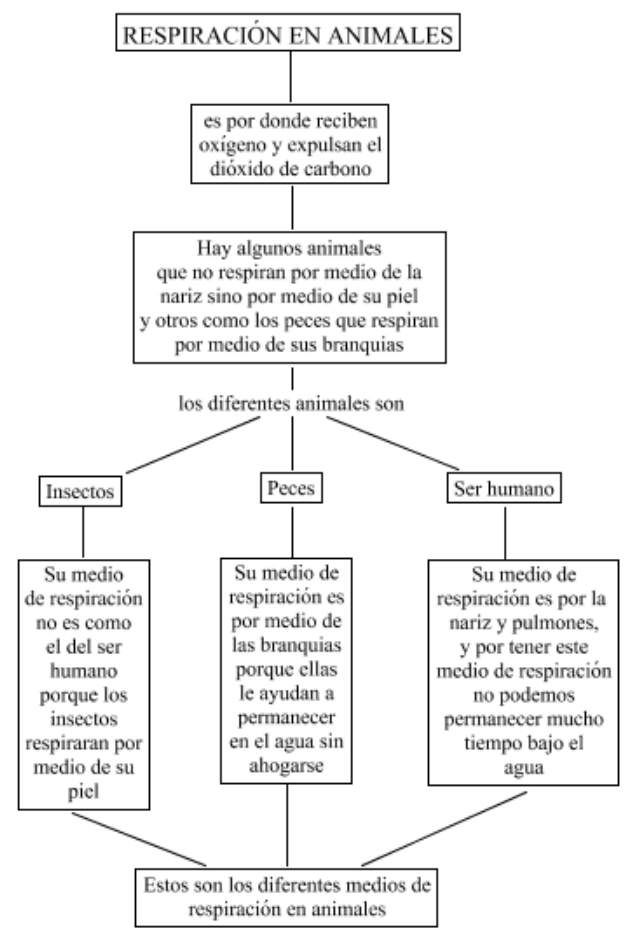

Figura 2. Cuestionario para la recolección de ideas previas acerca del concepto de respiración animal, adaptación de Banet \& Núñez (1990). 
Bio - grafia. Escritos sobre la Biología y su Enseñanza. ISSN 2027

Edición Extraordinaria. p.p. 1645-1655

Memorias del VIII Encuentro Nacional de Experiencias en Enseñanza de la Biología y la Educación Ambiental. III Congreso Nacional de Investigación en Enseñanza de la Biología.

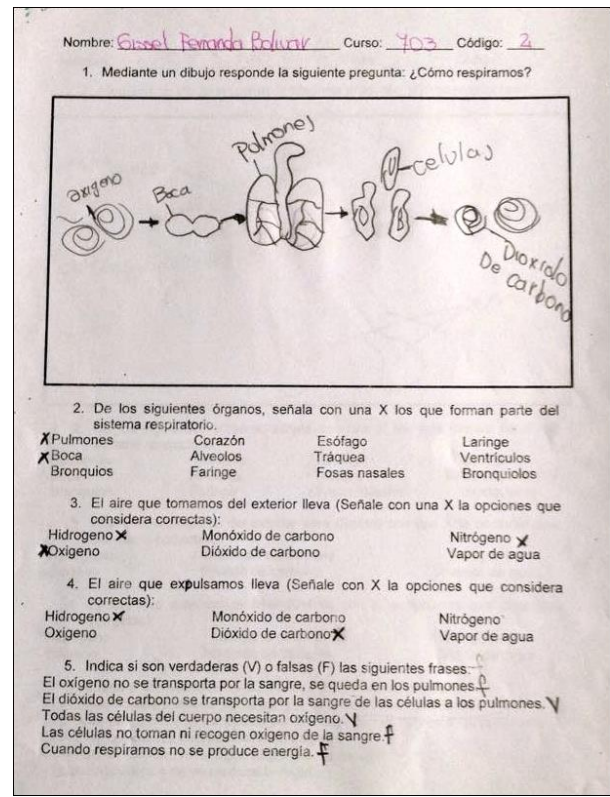

\section{Resultados y Discusión}

A través del análisis cualitativo sobre las ideas previas del concepto de respiración animal fue posible establecer un análisis de conglomerado (Figura 3), es decir, la agrupación de las palabras más utilizadas con un cierto tipo de similitud en las respuestas de los estudiantes; la frecuencia de los términos más referenciados por los estudiantes en los instrumentos (Figura 4) y la categorización de las ideas previas en los modelos explicativos presentados por Tamayo et al. (2008).

En primer instante se identifican relaciones muy estrechas entre los procesos antagónicos de respiración en plantas y animales, por ejemplo: -"Mediante el proceso de respiración los mamíferos toman el oxígeno, este se transporta por todo el cuerpo, en esto funciona el corazón que impulsa la sangre oxigenada por todas las células, después el oxígeno sale del cuerpo convertido en dióxido de carbono para que las plantas tomen ese dióxido de carbono y hagan el proceso de fotosíntesis y vuelva a hacer el mismo proceso". De la misma manera se observa una intencionalidad de nombrar estructuras que intervienen en el proceso pero desconocen su función, por ejemplo: -"El sistema respiratorio está compuesto por pulmones, boca, esófago, tráquea, fosas nasales y laringe... Entra oxigeno por la nariz, pasa el oxígeno por la tráquea, se procesa en los pulmones y sale dióxido de carbono por la boca". Por otro lado sustentamos la idea de los estudiantes acerca de la variabilidad de elementos (nitrógeno, vapor de agua, hidrogeno, oxigeno, dióxido y monóxido de carbono) que contiene el aire que tomamos del exterior en las consideraciones de Banet \& Nuñez (1990), en donde 
Bio - grafia. Escritos sobre la Biología y su Enseñanza. ISSN 2027

Edición Extraordinaria. p.p. 1645-1655

Memorias del VIII Encuentro Nacional de Experiencias en Enseñanza de la Biología y la Educación Ambiental. III Congreso Nacional de Investigación en Enseñanza de la Biología.

postulan que uno de cada cuatro alumnos piensan que el aire inspirado está formado solamente por oxígeno, ello pone de manifiesto que la inspiración o inhalación conlleva un proceso de selección de gases, de manera que a los pulmones sólo llega oxígeno.

Figura 3. Análisis de conglomerado en NVivo 10. Agrupación de palabras encontradas en los instrumentos de recolección de ideas previas acerca del concepto respiración animal con cierto grado de similitud.

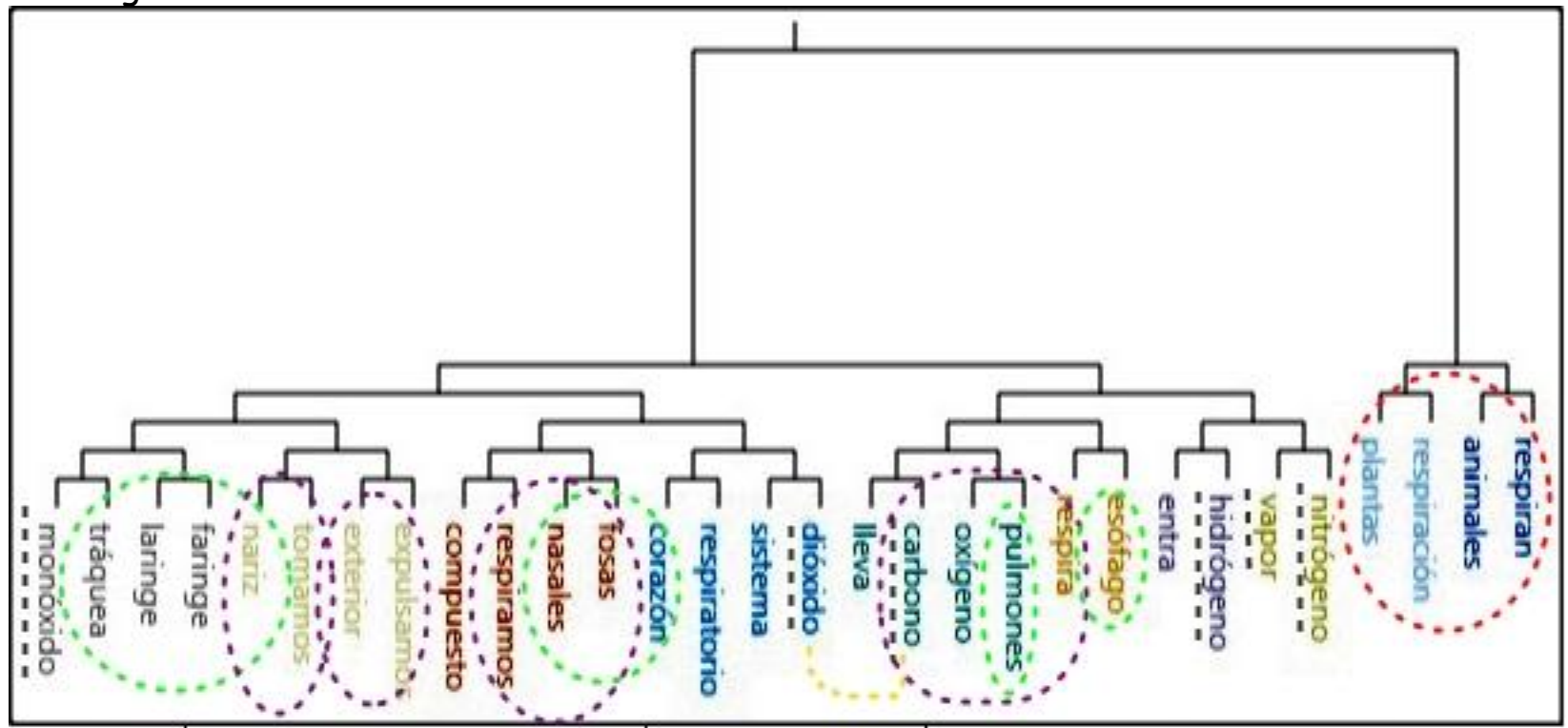

Los términos que más referencian los estudiantes están relacionados con estructuras relevantes en el proceso de respiración como los pulmones y las fosas nasales; y con el proceso de intercambio gaseoso, nombran la entrada de oxígeno y salida de dióxido de carbono. Lo anterior se sustenta en la aplicación del pre test del sistema respiratorio de Ochoa \& Camero (2005), en el cual las respuestas de los estudiantes reflejan que conocen algo de la respiración externa en el sentido de que se inhala aire con oxígeno y se exhala dióxido de carbono.

Figura 4. Frecuencia de los términos más referenciados por los estudiantes en la aplicación de los instrumentos de recolección de ideas previas acerca del concepto respiración animal. 
Bio - grafia. Escritos sobre la Biología y su Enseñanza. ISSN 2027

Edición Extraordinaria. p.p. 1645-1655

Memorias del VIII Encuentro Nacional de Experiencias en Enseñanza de la Biología y la Educación Ambiental. III Congreso Nacional de Investigación en Enseñanza de la Biología.

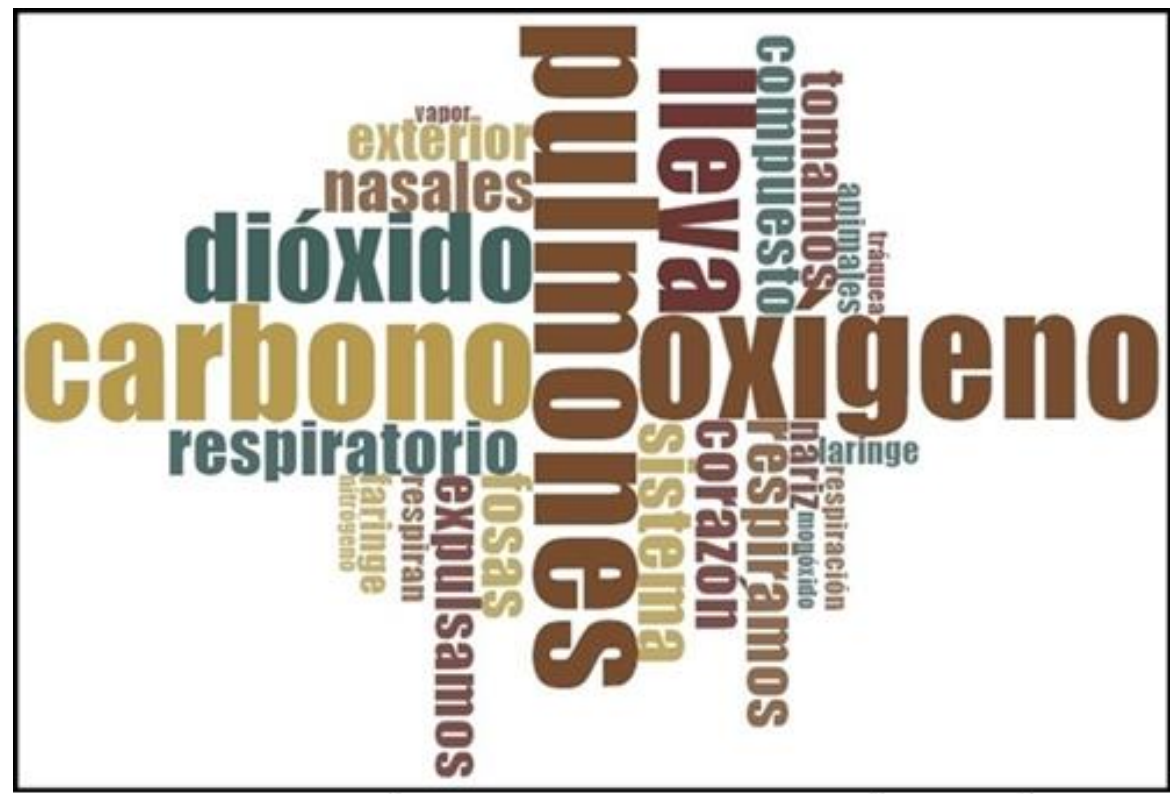

Por último, al categorizar las ideas previas que tienen los estudiantes acerca de la respiración animal (Tabla 1) con base a los modelos explicativos presentados por Tamayo et al. (2008), encontramos mayor tendencia en el modelo de Intercambio gaseoso, este modelo expresado por los estudiantes considera que el oxígeno se transforma en dióxido de carbono, es decir, se asume que el oxígeno respirado se transforma de manera directa en CO2. Los estudiantes asumen que en el intercambio de gases el oxígeno que entra al cuerpo se convierte en dióxido de carbono, el cual es eliminado como sustancia dañina para el organismo, le dan cierta facultad benéfica al oxígeno considerando que es bueno porque permite que los órganos y en general el organismo funcione adecuadamente (Tamayo et al., 2008). En el modelo pseudomolecular se categorizan las respuestas de los estudiantes en las cuales intentan elaborar explicaciones a nivel molecular del proceso de respiración pero de manera muy superficial, Tamayo et al., (2008) indican que se presenta gran dificultad para realizar explicaciones a nivel molecular. De la misma manera, podemos categorizar las ideas previas de los estudiantes en el modelo vitalista, este modelo explicativo plantea que el oxígeno es fuente de energía para el organismo. Según Tamayo et al., (2008), los estudiantes que emplean este modelo en sus explicaciones no plantean relaciones con la nutrición ni hacen explicaciones a nivel molecular.

Tabla 1. Categorización de las ideas previas de los estudiantes acerca de la respiración animal, con respecto a los modelos explicativos propuestos por Tamayo et al., (2008). 
Bio - grafia. Escritos sobre la Biología y su Enseñanza. ISSN 2027

Edición Extraordinaria. p.p. 1645-1655

Memorias del VIII Encuentro Nacional de Experiencias en Enseñanza de la Biología y la Educación Ambiental. III Congreso Nacional de Investigación en Enseñanza de la Biología.

\begin{tabular}{|c|c|c|c|}
\hline $\begin{array}{l}\text { Modelos } \\
\text { Explicativos } \\
\text { (Tamayo et } \\
\text { al., 2008) }\end{array}$ & Intercambio gaseoso & Pseudomolecular & Vitalista \\
\hline $\begin{array}{l}\text { Ideas Previas } \\
\text { Estudiantes }\end{array}$ & $\begin{array}{l}\text { E1.-"La función del sistema } \\
\text { respiratorio es recibir } \\
\text { oxígeno y botar dióxido de } \\
\text { carbono". } \\
\text { E2.-"La respiración en los } \\
\text { animales se da cuando } \\
\text { entra el oxígeno por las } \\
\text { fosas nasales y se expulsa } \\
\text { gas carbónico por la boca". } \\
\text { E3.-"Los animales respiran } \\
\text { oxígeno y botan dióxido de } \\
\text { carbono". } \\
\text { E4. -"La función de la } \\
\text { respiración en los animales } \\
\text { es ingresar oxígeno al } \\
\text { cuerpo y eliminar el dióxido } \\
\text { de carbono". }\end{array}$ & $\begin{array}{l}\text { E5. - "El ser humano } \\
\text { respira con ayuda de } \\
\text { proteínas y alimentos } \\
\text { que ayudan a } \\
\text { funcionar nuestros } \\
\text { pulmones". } \\
\text { E6. -"La respiración } \\
\text { es el proceso por el } \\
\text { cual los seres vivos } \\
\text { absorben oxígeno y lo } \\
\text { transportan por la } \\
\text { sangre a las células". } \\
\text { E7. -"..En la inhalación } \\
\text { el oxígeno crea una } \\
\text { oxidación y eso } \\
\text { genera la energía que } \\
\text { se utiliza". }\end{array}$ & 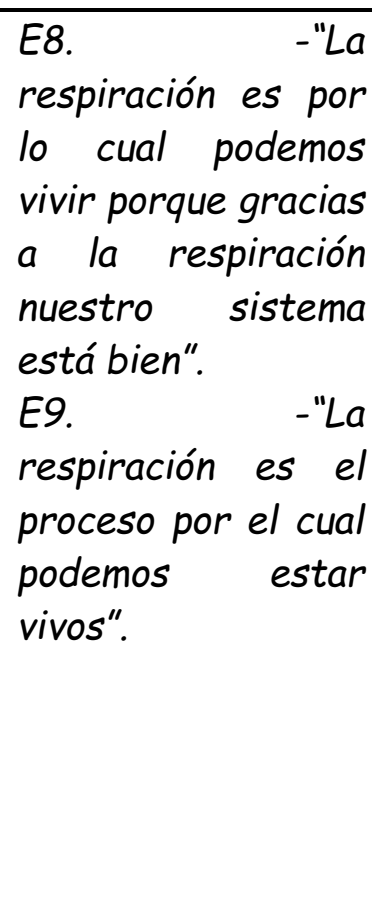 \\
\hline
\end{tabular}

Figura 5. Porcentaje de cobertura de las ideas previas acerca del concepto de respiración categorizadas en los modelos explicativos propuestos por Tamayo et al., (2008).

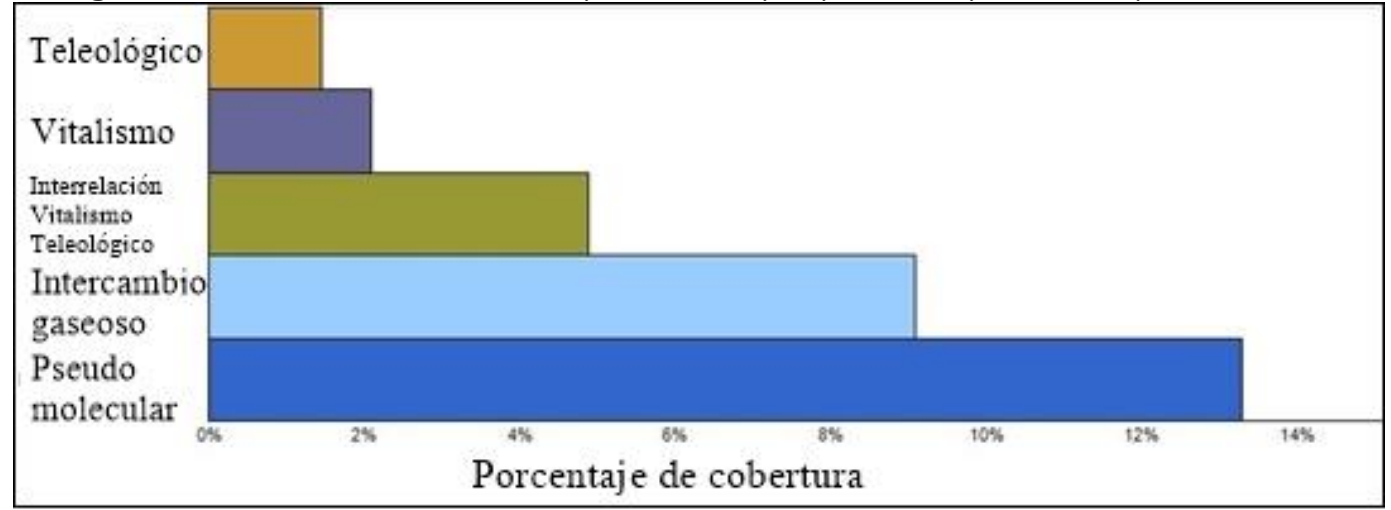


Bio - grafia. Escritos sobre la Biología y su Enseñanza. ISSN 2027

Edición Extraordinaria. p.p. 1645-1655

Memorias del VIII Encuentro Nacional de Experiencias en Enseñanza de la Biología y la Educación Ambiental. III Congreso Nacional de Investigación en Enseñanza de la Biología.

En la Figura 5 se evidencia el porcentaje de cobertura de las categorías planteadas en referencia a los instrumentos analizados, es importante destacar que los modelos explicativos postulados por Tamayo et al. (2008) tan solo cubren el $30 \%$ de las ideas previas en este caso, esto se debe a que los estudiantes se limitan a referenciar algunos órganos y partes del sistema respiratorio para explicar el proceso. Por ello, se propone una categoría emergente, la categoría de "Órganos y estructuras" que agruparía respuestas de este tipo: -"Se respira por la nariz y llega a los pulmones"; -"Los seres humanos respiramos por los pulmones"; -"Se respira por la nariz, llega a los pulmones y corazón". En tal grupo se ubican las estructuras y órganos relacionados al sistema respiratorio referenciadas en las ideas previas de los estudiantes, claro que con una función poco detallada dentro del proceso.

\section{Conclusiones}

Las ideas previas de los estudiantes acerca del concepto de Respiración Animal presentan cierta tendencia al referirse a un proceso de intercambio gaseoso, hacen mención a la entrada de oxígeno y salida de dióxido de carbono, ya sea por la piel, branquias, nariz, boca - pulmones, dependiendo de la clase de animal citada. En los instrumentos se observan dificultades para explicar procesos a nivel celular, y la asociación de estructura-función; de la misma manera, se evidencia la importancia de la respiración en los seres vivos.

Al identificar ideas previas categorizadas o no en modelos explicativos comunes se debe saber cómo utilizarlas, es decir, el maestro debe partir de dichas ideas previas para la explicación y construcción del concepto, permitiéndole a los estudiantes crear una conexión entre la información que se está aprendiendo y la que se tenía con anterioridad. Por ello, la caracterización y categorización de las ideas previas del grupo de estudio constituirán un marco de referencia importante para planificar el concepto a desarrollar, las actividades, estrategias, instrumentos y la evaluación que establecerán el proceso de enseñanza.

A través de este tipo de investigaciones es posible conocer, comprender y categorizar las ideas previas de los estudiantes sobre un concepto específico, esto con el propósito de diseñar e implementar unidades didácticas o cualquier otro tipo de propuesta de trabajo en el proceso de enseñanza-aprendizaje. En este caso, categorizar las ideas previas resulta ser una acción significativa al momento de planear y diseñar la unidad didáctica; de igual manera, en la enseñanza de las ciencias aumenta su importancia si se pretende revisar la evolución del concepto en los estudiantes después de la aplicación. 
Bio - grafia. Escritos sobre la Biología y su Enseñanza. ISSN 2027

Edición Extraordinaria. p.p. 1645-1655

Memorias del VIII Encuentro Nacional de Experiencias en Enseñanza de la Biología y la Educación Ambiental. III Congreso Nacional de Investigación en Enseñanza de la Biología.

\section{Bibliografía}

Ausubel, D. 1968. Educational Psichology: A cognitive view. (Rinehart y Winston. New York).

Ausubel, D., Novak, J., \& Hanesian, H. 1983. Psicología Educativa: Un punto de vista cognoscitivo. $2^{\circ}$ Edición. Editorial TRILLAS. México.

Banet, E., \& Nuñez, F. 1990. Esquemas conceptuales de los alumnos sobre la respiración. Revista Enseñanza de las Ciencias, 8 (2), pp. 105-111.

Díaz B., F., \& Hernández, G. 1999. Estrategias docentes para un aprendizaje significativo. McGraw Hill. México, 232p.

Driver, R., Guesne, E., \& Tiberghien, A. 1985. Children's ideas in science. Milton Keynes, England: Open University Press.

Driver, R. 1986. Psicología cognoscitiva y esquemas conceptuales de los alumnos. Revista Enseñanza de las Ciencias, Ciencias, Vol. 7(1), pp. 35-44. Vol. 4(1), pp. 3-15.

García Hourcade, J. L., \& Rodríguez De Ávila, C. 1988. Ideas previas, esquemas alternativos, cambio conceptual y el trabajo en el aula. Revista Enseñanza de las ciencias, 6 (2), 161-166.

Giordan, A. 1982. La enseñanza de las Ciencias. (Pablo del Rlo: Madrid).

González García, F. M. 1992. Los mapas conceptuales de J.D. Novak como instrumentos para la investigación en didáctica de las ciencias experimentales. Revista Enseñanza de las ciencias, 10 (2), 148-158.

Gutiérrez, R. 1987. Psicología y aprendizaje de las ciencias. El modelo de Ausubel. Revista Enseñanza de las ciencias, 5 (2), 118-128.

Nuñez, F., \& Banet, E. 1996. Modelos conceptuales sobre las relaciones entre digestión, respiración y circulación. Revista Enseñanza de las Ciencias. 14, 3, 261-278. 
Bio - grafia. Escritos sobre la Biología y su Enseñanza. ISSN 2027

Edición Extraordinaria. p.p. 1645-1655

Memorias del VIII Encuentro Nacional de Experiencias en Enseñanza de la Biología y la Educación Ambiental. III Congreso Nacional de Investigación en Enseñanza de la Biología.

Ochoa de Toledo, M., \& Camero, R. E. 2005. Aplicación y evaluación de una Unidad Didáctica sobre el sistema respiratorio. Revista de Investigación N 57, 143-164.

Rodríguez, M. L., Moreira, M. A., Caballero, M.C., Greca, I. 2008. La teoría del aprendizaje significativo en la perspectiva de la psicología cognitiva. Ediciones Octaedro, S.L. Barcelona.

Tamayo A., O. 1999. Tendencias sobre el concepto de bioenergética en estudiantes de primero de bachillerato. Sus representaciones mentales. Trabajo de investigación para optar al título de Máster. Barcelona: Universidad Autónoma de Barcelona.

Tamayo, O., Orrego, M. y Dávila, A. 2008. "Modelos explicativos del concepto de respiración". Memorias CIIEC 2008, No. 3, Vol. 2, pp. 50-63. 